
\title{
$\begin{array}{ll}\text { Research Square } & \begin{array}{l}\text { Preprints are preliminary reports that have not undergone peer review. } \\ \text { They should not be considered conclusive, used to inform clinical practice, } \\ \text { or referenced by the media as validated information. }\end{array}\end{array}$
}

\section{Application of droplet digital PCR to detect human Alu DNA elements in biodistribution assays in pre-clinical studies using rodents}

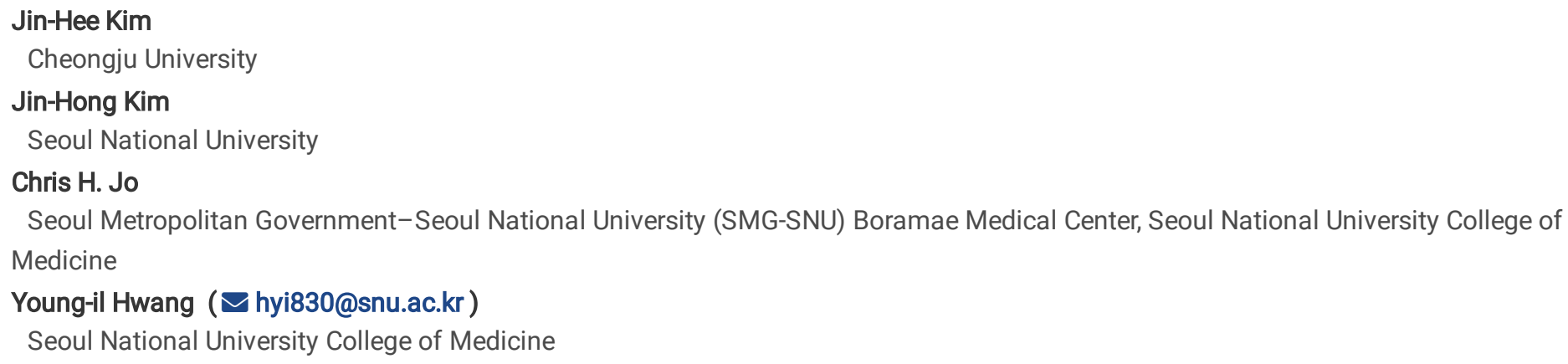

\section{Research Article}

Keywords:

Posted Date: February 10th, 2022

DOI: https://doi.org/10.21203/rs.3.rs-1259115/v1

License: (9) (1) This work is licensed under a Creative Commons Attribution 4.0 International License. Read Full License 


\section{Abstract}

Biodistribution and engraftment assays for administered human cells in animal organs must be performed to ensure the safety and efficacy of the human cells in preclinical studies. Quantitative real-time polymerase chain reaction (qRT-PCR) is currently the standard method used to detect Alu DNA elements. The recently developed droplet digital PCR (ddPCR) method has been adopted in many biological fields, in which the small quantities of DNA must be detected. In this study, we evaluated whether ddPCR could replace qRT-PCR in biodistribution research. We applied ddPCR to detect human Alu DNA elements using various concentrations of genomic DNA (gDNA) extracted from human mesenchymal stem cells spiked with $100 \mathrm{ng}$ of rat gDNA. We found that ddPCR could specifically detect human DNA and exhibited linearity within a concentration range of $10 \mathrm{ag}-100 \mathrm{pg} / 20 \mu \mathrm{l}$. ddPCR was far more sensitive than qRT-PCR in measuring Alu elements, with a reliable lower limit of detection of $100 \mathrm{ag} \mathrm{DNA} / 20 \mu \mathrm{l}$ reaction. Additionally, the copy numbers of Alu elements were quantified using ddPCR in an ex vivo study examining DNA samples extracted from mouse spleen and lymph nodes that had been spiked with human natural killer cells; no human DNA was detected using qRT-PCR in this assay. Thus, we established a ddPCR method for detecting human gDNA in bio-distribution assays of human cells in rodents in preclinical studies.

\section{Introduction}

In recent years, different types of ex vivo expanded autologous or allogenic cells, such as natural killer (NK) cells ${ }^{1}$ and chimeric antigen receptor-engineered T-cells ${ }^{2}$, have been applied clinically as a form of cell therapy. Mesenchymal stem cells (MSCs) in particular have been widely applied as a treatment module for regenerative medicine and chronic inflammatory diseases ${ }^{3}$. In pre-clinical studies, the biodistribution, engraftment, and persistence of human cells within animal models in which the cells are implanted must be evaluated ${ }^{4,5}$, as well as the safety and activity of the cells ${ }^{4,6}$.

To evaluate the bio-distribution of administered MSCs in experimental animals, many in vivo and ex vivo methods have been developed ${ }^{7}$. Methods for the ex vivo detection of human cells in animal tissues include immunohistochemical staining and in situ hybridization; however, the most sensitive and accurate method is to detect human DNA markers within animal tissues such as Alu elements, primatespecific short interspersed elements ${ }^{8}$, for which quantitative real-time polymerase chain reaction (qRT-PCR) is the standard detection method $^{5,9-11}$.

Although qRT-PCR is a powerful technique that enables the accurate and sensitive quantification of specific DNA sequences, there are some technical disadvantages. For example, external standard calibration curves based on reference standard materials (RSMs), which are timeconsuming to generate and maintain, must be generated to quantify qRT-PCR signals for each experiment. Additionally, the cycle threshold $(\mathrm{Ct})$ value may vary depending on the structure of the RSM. The secondary structure of reference plasmid DNA can affect qRT-PCR titration assays. For example, the supercoiled conformation of circular plasmid DNA suppresses primer annealing to the DNA template, resulting in regression line shift and titer overestimation ${ }^{12}$. Furthermore, the sensitivity of qRT-PCR is affected by various PCR inhibitors ${ }^{13}$.

Recently, a new highly sensitive DNA detection system, digital PCR (dPCR), has been developed ${ }^{14}$. In contrast to qRT-PCR, target DNA sequences are directly quantified in dPCR without the need to generate a standard curve using an RSM. This method is highly sensitive and precise, and is thus applied in many biological fields for the detection of rare DNA sequences such as microRNAs in clinical samples ${ }^{15}$, rare mutations in cell-free DNA samples ${ }^{16}$, chromosome abnormalities ${ }^{17}$, and loaded pathogens such as virus and bacteria ${ }^{18}$. However, dPCR has not yet been applied to identify Alu elements in biodistribution studies of human cells administered into experimental animals.

Droplet digital PCR (ddPCR) ${ }^{19}$ is a recently developed and commonly used dPCR method, in which the PCR reaction is performed in numerous nanoliter volume lipid droplets. In the present study, we evaluated the performance of ddPCR for identifying and quantifying Alu elements in biodistribution studies of human cells in experimental animals.

\section{Results}

Specificity First, we examined the specificity of ddPCR for the Alu element and evaluated its ability to differentiate between human DNA (hDNA) and matrix rodent DNA. We compared the ddPCR results of the NC and NTC groups to those of the experimental group, which contained $100 \mathrm{ng}$ of human Wharton's jelly-derived MSC DNA and $100 \mathrm{ng}$ of rat DNA per reaction mixture. A high Alu DNA copy number (1.8 $\times 10^{4}$ copies/total reaction mixture) was detected in the experimental group, while negative results (0-0.03 copies/total reaction mixture) were obtained in both the NC and NTC groups (Fig. 1), indicating that no false-positive results were generated by ddPCR. 
Detection limit Next, we determined the detection limit of the ddPCR method for identifying Alu elements. We calculated the dose range of hDNA in $20 \mu \mathrm{l}$ of reaction mixture containing $100 \mathrm{ng}$ of rat DNA. A single copy of the human genome has an average mass of $6.46 \mathrm{pg}^{20}$ and contains approximately $1,090,000$ copies of $A / u$ elements ${ }^{21}$. Therefore, one Alu element copy weighs 5.9 attograms (ag), and 10 ag of hDNA contains 1.69 copies of Alu elements. Based on this calculation, hDNA was serially diluted and added to the ddPCR mixture at concentrations ranging from $10^{-1}$ to $10^{4} \mathrm{ag} / 20 \mu \mathrm{l}$. Each mixture also contained $100 \mathrm{ng}$ of rat DNA. For the ddPCR mixtures containing 10$10^{4} \mathrm{ag} / 20 \mu \mathrm{hDNA}, 1.4,9.2,104$, and 1024 copies of Alu elements were detected by ddPCR, respectively (Figure 2A). These values displayed linearity and strong correlation $\left(R^{2}=0.9974\right)$. The Alu element copy number detected in the $10 \mathrm{ag} / 20 \mu$ concentration ddPCR mixture $(1.4$ copies/total reaction mixture) closely matches that calculated theoretically for the same hDNA concentration (1.69); this suggests that the copy number obtained via ddPCR is reliable. However, because the 1 and $10^{-1}$ at/20 $\mu \mathrm{l}$ mixtures measured as 1.4 and 1.2 copies/total reaction mixture, respectively, the limit of reliable values is thought to be 9.2 copies of $100 \mathrm{ag}$.

Linearity The results of biodistribution assays must be linear ${ }^{22,23}$. As shown in Figure 2 , the ddPCR results were linear within the range of $10^{-1}-10^{4} \mathrm{ag} / 20 \mu \mathrm{l}$. To expand this range, we performed ddPCR using 10 -fold dilutions of hDNA at concentrations between $1 \mathrm{fg} / 20 \mu \mathrm{l}$ and $10^{2} \mathrm{ng} / 20 \mu \mathrm{l}$ (Fig. 3). The results were linear across concentrations from $1 \mathrm{fg} / 20 \mu \mathrm{l}$ to $10^{2} \mathrm{pg} / 20 \mu \mathrm{l}\left(\mathrm{R}^{2}=0.9397\right)$. Taken together, our results indicate that ddPCR exhibited linearity over a broad range of concentrations, from 10 ag to $100 \mathrm{pg} / 20 \mu \mathrm{l}$, for the detection of $A / u$ elements (Figures 2,3).

Comparison with qRT-PCR data Next, we compared our ddPCR results with those obtained by qRT-PCR, the method currently recommended for biodistribution assays. We generated qRT-PCR Ct values for Alu elements amplification. Due to potential matrix effects driven by xenogeneic DNA, we performed the qRT-PCR reactions both in the absence (Fig. 4A) and presence (Fig. 4B) of rat DNA.

In the absence of rat DNA, the qRT-PCR Ct values were linear within an hDNA concentration range of $10 \mathrm{fg}-100 \mathrm{pg} / 20 \mu \mathrm{l}(\mathrm{Fig} .4 \mathrm{~A})$; the Ct value at $10 \mathrm{fg} / 20 \mu \mathrm{l}$ was 30.95. In the presence of rat DNA, the Ct values were linear within a range of $10 \mathrm{fg}-10 \mathrm{pg} / 20 \mu \mathrm{l}(\mathrm{Fig}$. 4B); the Ct value at $10 \mathrm{fg} / 20 \mu \mathrm{l}$ was 33.95 , which was higher than that in the absence of rat DNA. This higher Ct value, by over 3 , was observed al all concentrations of hDNA (Fig. 4C, Table 1), revealing that the spiked rat DNA exerted a matrix effect.

To determine whether rat DNA also exerted an inhibitory effect during ddPCR, ddPCR was performed using an hDNA concentration range of $10^{1}-10^{4} \mathrm{fg} \mathrm{DNA} / 20 \mu \mathrm{l}$ in the absence (Fig. 4D) and presence (Fig. 4E) of $100 \mathrm{ng}$ rat DNA; the detected copy numbers for both sample types were similar.

Ex vivo application The results of our in vitro experiments suggested that ddPCR is an effective method to evaluate biodistribution. Therefore, we tested the ddPCR method ex vivo using SCID mice that were intravenously injected with human NK cells and sacrificed after 10 days. First, the spleen and inguinal nodes were harvested, paraffin-embedded, and immunostained for the hNK cell marker CD56. As shown in Figure 5 (left panel), a few CD56-positive cells were identified in both organs. We then performed ddPCR using $100 \mathrm{ng}$ of tissue DNA. The Alu element copy numbers calculated by ddPCR were 636 and 128 copies/100 ng of gDNA for the spleen and lymph nodes, respectively (right panel). However, qRT-PCR failed to detect hDNA in either organ (data not shown).

\section{Discussion}

In this study, we explored the effectiveness of ddPCR for the detection of Alu elements in biodistribution studies, and for detecting small quantities of hDNA in animal tissues, which are requirements for pre-clinical studies. Our results indicated that ddPCR met the specificity and linearity requirements for DNA detection. Notably, the detection limit of ddPCR was significantly lower than that of qRT-PCR. We then applied ddPCR to detect hDNA in SCID mouse organs following intravascular injection with hNK cells and were able to detect Alu elements that were not detected by qRT-PCR.

Prior to ddPCR, the template DNA was digested with the restriction enzyme Msel, an endonuclease that recognizes the sequence 5'-TTAA-3' and cleaves between the two T residues ${ }^{24}$. The theoretical probability of the TTAA sequence occurring throughout the human genome is $4^{-4}$ $(1 / 256)$. Because the human genome size is approximately $6.32 \mathrm{~Gb}^{20}$, the theoretical number of fragments yielded by Msel is $24,687,547$, far exceeding the total number of $A / u$ elements in the human genome, which is $1,090,000^{21}$. This suggests that Msel treatment caused the Alu elements to be distributed into the lipid droplets much more evenly, even when the hDNA concentrations within the samples were extremely low. Therefore, this improved the precision of the experiment.

Previous studies comparing ddPCR to qRT-PCR revealed that ddPCR was more precise and accurate, both within and between assays, as well as between different laboratories ${ }^{19,25-27}$. Regarding the sensitivity and limit of detection, the results of previous studies were dependent 
on the detection target and sample source, with some studies suggesting that ddPCR is less sensitive compared to qRT-PCR ${ }^{25,28}$. However, in more recent studies, the sensitivity of ddPCR has been reported to be higher than that of qRT-PCR. In a study measuring Bifidobacterium and Lactobacillus 16S RNA gene copy numbers in breast milk, ddPCR was 10-fold more sensitive than qRT-PCR ${ }^{29}$. Furthermore, ddPCR was 100-fold more sensitive than qRT-PCR when detecting the microRNA miR-29a in urine ${ }^{27}$. In the present study, the limit of detection was between 9.2 copies at 100 ag and 1.4 copies at 10 at, which is 1000 -fold lower than the $10 \mathrm{fg}$ limit of detection obtained with qRT-PCR. This was similar to the previous finding that ddPCR is 1000-fold more sensitive that qRT-PCR when detecting FAdV-4 virus contaminants in various samples including in vaccines ${ }^{30}$. Additionally, the Zika virus standard plasmid could be detected by ddPCR to a detection limit of 1 copy/ $\mu$ l, which was not detectable with qRT-PCR ${ }^{31}$.

In our ex vivo experiment, 31.8 and 6.4 copies of $A / u$ elements per $5 \mathrm{ng}$ DNA/ $\mu$ l were detected in DNA samples extracted from the spleen and lymph nodes of mice injected with human NK cells, respectively. This suggested that $3.77 \mathrm{ag}(31.8 \times 20 \times 6.46 / 1,090,000)$ and 0.76 ag $(6.4 \times 20 \times 6.46 / 1090000)$ of hDNA were present in the 100 ng DNA per PCR mixture for the spleen and lymph node samples, respectively. Given that the detection limit for qRT-PCR was $10 \mathrm{fg}$ of hDNA, this explains why we were unable to detect hDNA using qRT-PCR in our ex vivo experiment; the NK cell hDNA observed in the tissue sections was diluted with mouse DNA to a concentration below the detection limit of qRT-PCR. Or, the qRT-PCR sensitivity in our laboratory might not be enough. Previously reported qRT-PCR sensitivities include 1 pg DNA/reaction ${ }^{32}, 0.2$ pg DNA $^{33}$, and 0.1-0.01 pg DNA ${ }^{10}$. The lowest reported detection limit is $0.01 \mathrm{pg}^{9}$. Even with this level of sensitivity, hDNA could not be detected in our DNA samples extracted from the lymph nodes.

Currently, we do not have criteria to define how many human cells would be meaningfully interpreted as being present in animal organs in preclinical studies, and further studies are needed to develop such criteria.

In our qRT-PCR experiments, the Ct values were higher in the presence of rat DNA (Figure 4C). A similar phenomenon has been observed in the presence of a high amount of porcine DNA ${ }^{32}$. It is not currently clear why the presence of xenogeneic DNA reduces the amplification efficiency of hDNA. One possible explanation is the presence of DNA inhibitors in the PCR mixture; various substances can act as PCR inhibitors $^{13}$. This phenomenon did not occur in the ddPCR experiments, which are not affected by PCR inhibitors ${ }^{35,36}$. This is because reductions in signal driven by PCR inhibitors do not affect the binary positive/negative readings for each micro-well in ddPCR. This highlights another advantage of the ddPCR method compared to qRT-PCR.

\section{Conclusion}

In this study, we developed and validated a highly sensitive and specific ddPCR system to study hDNA biodistribution in rodent gDNA mixtures. ddPCR could potentially replace the present standard detection method, qRT-PCR, due to its higher sensitivity, lower sensitivity to PCR inhibitors, and the lack of requirements for RSMs.

\section{Methods}

Human mesenchymal stem cells (hMSCs) Wharton's jelly-derived MSCs were developed as previously described ${ }^{37}$ and maintained in lowglucose DMEM (Hyclone, South Logan, UT, USA) supplemented with 10\% fetal bovine serum (Hyclone), $100 \mathrm{U} / \mathrm{mL}$ penicillin, 100 $\mathrm{gg} / \mathrm{mL}$ streptomycin, and $0.25 \mu \mathrm{g} / \mathrm{mL}$ amphotericin (Welgene Inc., Gyeongsan, Korea). The study protocol was approved by the Institutional Review Board of Seoul National University (SNU-J-1511-005-715).

Human NK cells Human NK (hNK) cells were kindly gifted by Chaok Yim (S Bio Co. Ltd.). The hNKs were immediately injected intravenously into 6-7-week-old NOG mice without any maintenance.

Animals Adult Sprague-Dawley rats were purchased from Koatech (Pyeongtaek, Korea). The animals were kept at the animal facility of Seoul National University College of Medicine under specific pathogen-free conditions. All animal experiments were approved by the IACUC of Seoul National University (SNU-151228-4-1). For rat DNA preparation, rats were anesthetized with Zoletil (Virbac, intraperitoneally, 30 $\mathrm{mg} / \mathrm{kg}$ ) and Rompun (Bayer Korea, intraperitoneal, $10 \mathrm{mg} / \mathrm{kg}$ ). Following anesthesia, organs such as the liver, pancreas, testis, and brain were harvested and immediately subjected to DNA extraction as described below.

NOG mice (6-7 weeks old) were purchased from Koatech (Pyeontaek). The animals were kept at the animal facility of the laboratory animal center at the Osong Medical Innovation Foundation (KBIO) under specific pathogen-free conditions. All animal experiments were approved by Osong Medical Innovation Foundation (KBIO-IACUC-2021-163-1). Twenty million hNK cells were injected intravenously into the NOG mice, which were sacrificed 10 days after injection. 
DNA extraction DNA was extracted from twelve types of rodent organ. Genomic DNA (gDNA) was extracted using a DNeasy Blood \& Tissue Kit (QIAGEN, Hilden, Germany) following the manufacturer's protocol. The gDNA concentration was determined using a Thermo Scientific NanoDrop One spectrophotometer (Thermo Scientific Inc., Waltham, USA). DNA samples were kept at $-80^{\circ} \mathrm{C}$ until use.

qRT-PCR qRT-PCR to detect Alu elements as a human-specific marker was performed using a Thermo Scientific QuantStudio Real-Time System. The following primers were synthesized (Bioneer, Daejeon, Korea) and used in the qRT-PCR reaction: human Alu, YB8-ALU-S68: 5'GTCAGGAGATCGAGACCATCCT-3' (position 68-90) and YB8-ALU-AS244: 5'-AGTGGCGCAATCTCGGC-3' (position 244-227) ${ }^{38}$. All TaqMan assays were performed using the following PCR conditions: denaturation at $95^{\circ} \mathrm{C}$ for $5 \mathrm{~min}$, followed by 40 cycles of denaturing at $95^{\circ} \mathrm{C}$ for $30 \mathrm{~s}$ and annealing at $60^{\circ} \mathrm{C}$ for $20 \mathrm{~s}$. Reactions were performed in duplicate. We used PowerUp SYBR Green Master Mix (Applied Biosystems, Cat No a25741) as the fluorescent reporter system for qRT-PCR instead of the FAM-probe, which we used in ddPCR; a pilot study revealed that the results obtained using both fluorescent reporter systems for qRT-PCR were equivalent (data not shown).

ddPCR ddPCR was performed on a QX200 AutoDG Droplet Digital PCR System (Bio-Rad, Hercules, CA, USA). The same primers were used for ddPCR as those used for qRT-PCR. The following probe was used for ddPCR: YB8-ALU-167, 5'-FAM-AGCTACTCGGGAGGCTGAGGCAGGATAMRA-3' (position 167-192) ${ }^{38}$. PCR was performed using the following amplification conditions: $10 \mathrm{~min}$ at $95^{\circ} \mathrm{C}, 40 \mathrm{cycles}$ of $30 \mathrm{~s}$ at $95^{\circ} \mathrm{C}$ and $60 \mathrm{~s}$ at $56^{\circ} \mathrm{C}$, and then $10 \mathrm{~min}$ at $98^{\circ} \mathrm{C}$. Amplified DNA was stored at $4^{\circ} \mathrm{C}$. Each ddPCR analysis batch contained a set of samples, negative template control (NTC), which contained mixture, probes, primers, and $100 \mathrm{ng}$ of gDNA extracted from rat liver, and negative control (NC), which contained mixture, probes, primers, and diethylpyrocarbonate-treated water. DNA extracted from human cells was spiked into rat liver DNA. All DNA samples were treated with the restriction enzyme Msel (1 unit/20 $\mu$ l) prior to droplet generation, which did not cut the primers, probe, or Alu sequence. This was to reduce the potential increase in mixture viscosity of the mixture caused by the large amount of DNA present (>100 ng DNA), which could influence the average volume of the droplets ${ }^{19}$. The PCR products were transferred to a QX200 Droplet Reader for analysis. The ddPCR data were analyzed using QX200 Droplet Reader control software QuantaSoft 1.7.4 (BioRad, Hercules, CA, USA) and presented as copy number/ $\mu$ l.

Histology Severe combined immunodeficient (SCID) mice were intravenously administered with 20 million hNK cells and then sacrificed 10 days post-injection. The spleen and inguinal lymph nodes were harvested, fixed in $4 \%$ paraformaldehyde solution, and embedded in paraffin. Tissue sections of $4 \mu \mathrm{l}$ thickness were sliced, mounted on glass slides, and subjected to immunohistochemical staining to detect human CD56 (NK cell marker). Before staining, the sections were incubated in Antigen Retriever Citrate Buffer, pH 6.0 (Sigma, Catalog \# C9999) for $20 \mathrm{~min}$ at $100^{\circ} \mathrm{C}$. Non-specific antibody binding was blocked by incubating the sections with $15 \%$ normal goat serum (Gibco, Dublin, Ireland) in phosphate buffered saline for $1 \mathrm{~h}$ at room temperature. Sections were incubated with anti-human CD56 antibody (1:100 dilution, clone 123C3.D5, Cell Marque, Cat. No. 156M-85, Rocklin, CA, USA) overnight at $4^{\circ} \mathrm{C}$, then with anti-mouse-HRP antibodies (1:200 dilution, Sigma, Cat. No. A9044-2ML) for $1 \mathrm{~h}$ at room temperature. The final staining step was performed using 3,3'-diaminobenzidine solution. Sections were counter-stained with hematoxylin and then mounted with Vector mounting medium (Vector Labs).

Data analysis DNA copy numbers detected by ddPCR were calculated using QuantaSoft version 1.7.4 (Bio-Rad, Hercules, CA, USA) software. Linear regression curves for the DNA concentrations measured by ddPCR and the Ct values obtained by qRT-PCR were calculated using GraphPad Prism Version 6 (GraphPad Prism Software Inc., San Diego, CA, USA).

\section{Data availability}

The data of the present study are available from the corresponding author on request.

\section{References}

1. Kim, J. M. et al. Adjuvant therapy using ex vivo-expanded allogenic natural killer cells in hepatectomy patients with hepatitis B virus related solitary hepatocellular carcinoma: MG4101 study. Ann. Hepatobiliary Pancreat Surg. 25, 206-214 (2021).

2. Adami, A. \& Maher, J. An overview of CAR T-cell clinical trial activity to 2021. Immunother. Adv. 1:Itab004 (2021).

3. Wang, L. T. et al. Human mesenchymal stem cells (MSCs) for treatment towards immune- and inflammation-mediated diseases: review of current clinical trials. J. Biomed. Sci. 23, 76 (2016).

4. FDA Department of Health and Human Services. Guidance for industry: preclinical assessment of investigational cellular and gene therapy products, Docket Number: FDA-2012-D-1038. https://www.fda.gov/media/87564/download (2013). 
5. MFDS (Ministry of Food and Drug Safety). Considerations in Biodistribution Assessment of Stem Cell Therapy Product. (in Korean) https://www.mfds.go.kr/brd/m_1060/view.do?

seq $=12487 \& \operatorname{srchFr}=\& \operatorname{srchTo}=\& \operatorname{srchWord}=\& \operatorname{srchTp}=\& i t m \_s e q \_1=0 \& i t m \_s e q \_2=0 \&$ multi_itm_seq=0\&company_cd=\&company_nm=\&page=82 (2014)

6. MFSD (Ministry of Food and Drug Safety). Considerations in Quality. Non-clinical and Clinical Assessment of Stem Cell Therapy Product. (in Korean) https://www.nifds.go.kr/brd/m_158/view.do?

seq $=11723 \&$ srchFr=\&srchTo=\&srchWord=\&srchTp=\&itm_seq_1=0\&itm_seq_2=0\&multi_itm_seq=0\&company_cd=\&company_nm=\&page=1 (2014)

7. Wang, F. et al. Comparative strategies for stem cell biodistribution in a preclinical study. Acta. Pharmacol. Sin. 41, 572-580 (2020).

8. Rowold, D. J. \& Herrera, R. J. Alu elements and the human genome. Genetica 108, 57-72 (2000).

9. Funakoshi, K. et al. Highly sensitive and specific Alu-based quantification of human cells among rodent cells. Sci. Rep. 7, 13202 (2017).

10. Prigent, J. et al. Human Progenitor Cell Quantification After Xenotransplantation in Rat and Mouse Models by a Sensitive qPCR Assay. Cell Transplant. 24, 1639-1652 (2015).

11. Song, P. et al. Human genome-specific real-time PCR method for sensitive detection and reproducible quantitation of human cells in mice. Stem Cell Rev. Rep. 8, 1155-1162 (2012).

12. Hou, Y., Zhang, H., Miranda, L. \& Lin, S. Serious overestimation in quantitative PCR by circular (supercoiled) plasmid standard: Microalgal pcna as the model gene. PLoS One 5, e9545 (2010).

13. Schrader, C., Schielke, A., Ellerbroek, L. \& Johne, R. PCR inhibitors - occurrence, properties and removal. J. Appl. Microbiol. 113, 10141026 (2012).

14. Vogelstein, B. \& Kinzler, K. W. Digital PCR. Proc. Natl. Acad. Sci. U. S. A. 96, 9236-9241 (1999).

15. Hindson, C. M. et al. Absolute quantification by droplet digital PCR versus analog real-time PCR. Nat. Methods. 10, 1003-1005 (2013).

16. Tong, Y., Shen, S., Jiang, H. \& Chen, Z. Application of Digital PCR in Detecting Human Diseases Associated Gene Mutation. Cell Physiol. Biochem. 43, 1718-1730 (2017).

17. Debrand, E., Lykoudi, A., Bradshaw, E. \& Allen, S. K. A Non-Invasive Droplet Digital PCR (ddPCR) Assay to Detect Paternal CFTR Mutations in the Cell-Free Fetal DNA (cffDNA) of Three Pregnancies at Risk of Cystic Fibrosis via Compound Heterozygosity. PLoS One. 10, e0142729 (2015).

18. Salipante, S. J. \& Jerome, K. R. Digital PCR-an emerging technology with broad applications in microbiology. Clin. Chem. 66, 117-123 (2020).

19. Hindson, B. J. et al. High-throughput droplet digital PCR system for absolute quantitation of DNA copy number. Anal. Chem. 83, 86048610 (2011).

20. Piovesan, A. et al. On the length, weight and GC content of the human genome. BMC Res. Notes. 12, 106 (2019).

21 Lander, E. S. et al. International Human Genome Sequencing Consortium. Initial sequencing and analysis of the human genome. Nature 409, 860-921 (2001).

22. EMA (Eruopean Medicines Agency). Draft ICH guideline M10 on bioanalytical method validation.

2019. https://www.ema.europa.eu/en/documents/scientific-guideline/draft-ich-guideline-m10-bioanalytical-method-validation-step-

2b_en.pdf.

23. FDA Department of Health and Human Services (2018). Bioanalytical method validation: guidance for industry. Docket Number: FDA2013-D-1020. 2018. https://www.fda.gov/media/70858/download.

24. Morgan, R. D. Mse I, a unique restriction endonuclease from Micrococcus species which recognizes 5' T decreases TAA 3'. Nucleic Acids Res. 16, 3104 (1988). 
25. Te, S. H., Chen, E. Y. \& Gin, K. Y. Comparison of Quantitative PCR and Droplet Digital PCR Multiplex Assays for Two Genera of BloomForming Cyanobacteria, Cylindrospermopsis and Microcystis. Appl. Environ. Microbiol. 81, 5203-5211 (2015).

26. Tang, H., Cai, Q., Li, H. \& Hu, P. Comparison of droplet digital PCR to real-time PCR for quantification of hepatitis B virus DNA. Biosci Biotechnol Biochem. 80, 2159-2164 (2016).

27. Wang, C. et al. Droplet digital PCR improves urinary exosomal miRNA detection compared to real-time PCR. Clin Biochem. 67, 54-59 (2019).

28. Hayden, R. T. et al. Comparison of droplet digital PCR to real-time PCR for quantitative detection of cytomegalovirus. J. Clin. Microbiol. 51, 540-546 (2013).

29. Qian, L., Song, H. \& Cai, W. Determination of Bifidobacterium and Lactobacillus in breast milk of healthy women by digital PCR. Benef. Microbes. 7, 559-569 (2016).

30. Dong, G. et al. Development and evaluation of a droplet digital PCR assay for the detection of fowl adenovirus serotypes 4 and 10 in attenuated vaccines. J. Virol. Methods. 265, 59-65 (2019).

31. Hui, Y. et al. Micro-droplet Digital Polymerase Chain Reaction and Real-Time Quantitative Polymerase Chain Reaction Technologies Provide Highly Sensitive and Accurate Detection of Zika Virus. Virol. Sin. 33, 270-277 (2018).

32. Abellaneda, J. M. et al. Validation of a quantitative polymerase chain reaction method for human Alu gene detection in microchimeric pigs used as donors for xenotransplantation. Transplant. Proc. 47, 132-135 (2015).

33. Creane, M., Howard, L., O'Brien, T. \& Coleman, C. M. Biodistribution and retention of locally administered human mesenchymal stromal cells: Quantitative polymerase chain reaction-based detection of human DNA in murine organs. Cytotherapy 19, 384-394 (2017).

35. Verhaegen, B. et al. Comparison of Droplet Digital PCR and qPCR for the Quantification of Shiga Toxin-Producing Escherichia coli in Bovine Feces. Toxins (Basel) 8, 157 (2016).

36. Yuan, D. et al. Droplet digital PCR for quantification of PML-RARa in acute promyelocytic leukemia: a comprehensive comparison with real-time PCR. Anal. Bioanal. Chem. 411, 895-903 (2019).

37. Jo, C. H. et al. Fetal mesenchymal stem cells derived from human umbilical cord sustain primitive characteristics during extensive expansion. Cell Tissue Res. 334, 423-433 (2008).

38. van der Horst, E. H. et al. TaqMan-based quantification of invasive cells in the chick embryo metastasis assay. Biotechniques $\mathbf{3 7}$, $940-$ $942(2004)$.

\section{Declarations}

\section{Acknowledgements}

This research was supported by the Bio \& Medical Technology Development Program of the National Research Foundation (NRF) funded by the Ministry of Science, ICT \& Future Planning (NRF-2015M3A9E6028677) and by the Basic Science Research Program through the National Research Foundation of Korea (NRF) funded by the Ministry of Education (2019R1I1A3A01050978).

\section{Author contributions}

J.H.K. ${ }^{1}$ and Y.-I. H. raised the idea and designed the study. J.H.K. ${ }^{2}$ and C.H. J. discussed the study design. J.H.K. ${ }^{1}$ collected and analyzed the data. All authors are involved in data analysis. Y.-I. H. wrote the manuscript. J.H.K. ${ }^{1}$, J.H.K. ${ }^{2}$, and C.H. J. edited the manuscript and approved the final version.

\section{Competing interests}

The authors declare that there is no competing interest. 


\section{Table}

Table 1. Ct values of qRT-PCR in the presence and absence of rat DNA.

\begin{tabular}{llll}
\hline hDNA concentraion $(\mathrm{fg})$ & without rat DNA & with rat DNA & increases \\
\hline 1 & 33.32 & 34.25 & 0.93 \\
10 & 30.95 & 33.95 & 3.00 \\
\hline 100 & 27.26 & 31.02 & 3.76 \\
\hline 1,000 & 23.96 & 27.94 & 3.96 \\
10,000 & 20.45 & 24.07 & 3.62 \\
\hline 100,000 & 17.21 & 21.04 & 3.83 \\
$1,000,000$ & 26.64 & n.d. & n.d. \\
\hline
\end{tabular}

n.d. $=$ not determined

\section{Figures}

\section{Figure 1}

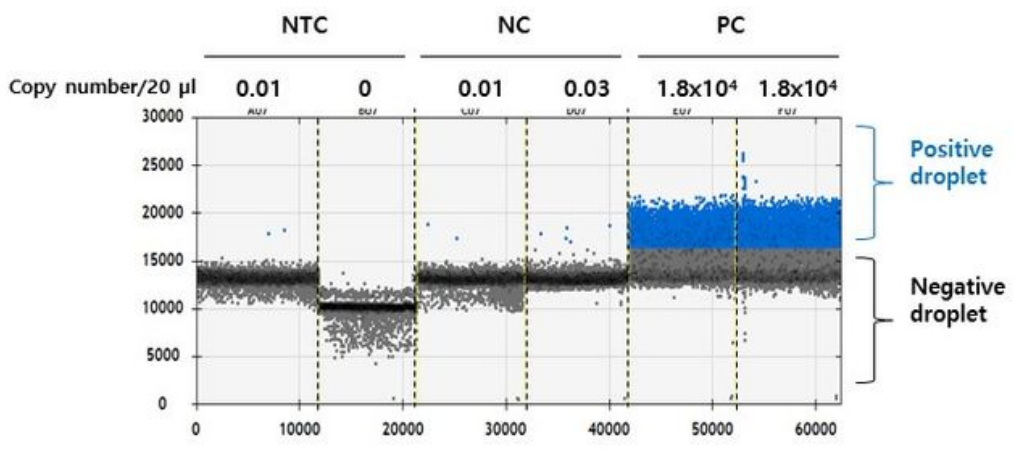

Figure 1

A representative profile of the ddPCR reactions showing the specificity of the ddPCR method for Alu elements. NTC, negative template control (no human DNA and $100 \mathrm{ng}$ of rat DNA); NC, negative control (no human DNA or rat DNA); PC, positive control (100 ng of human DNA and rat DNA). This experiment was repeated three times. 


\section{Figure 2}

A

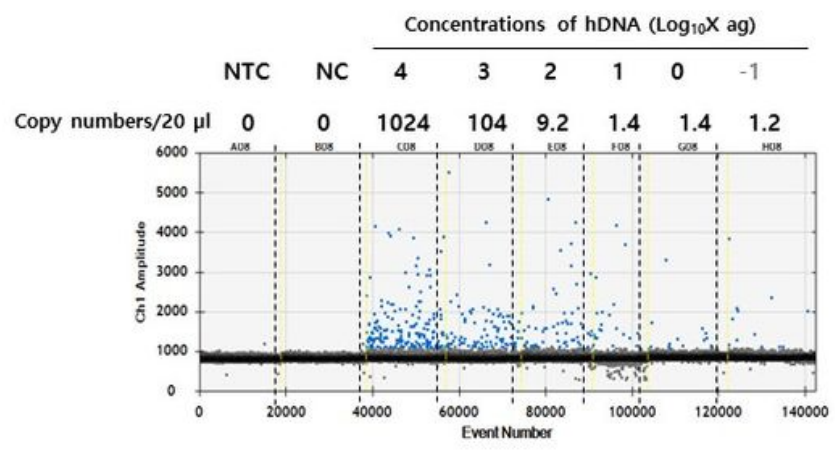

B

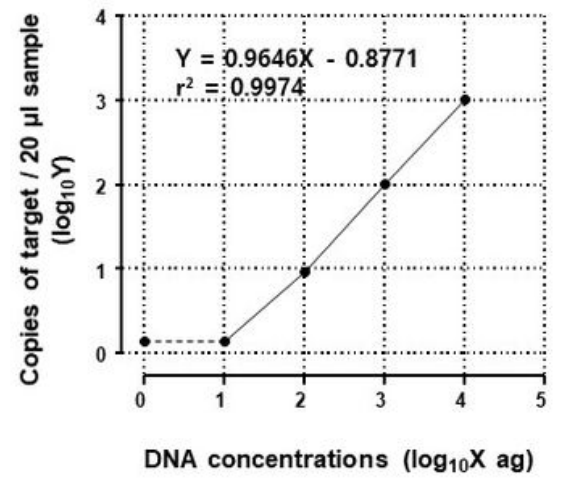

Figure 2

ddPCR reaction profiles at lower concentrations of hDNA. A representative reaction profile (A) and graph (B) are shown. NTC, negative template control (no human DNA and $100 \mathrm{ng}$ of rat DNA); NC, negative control (no human DNA or rat DNA); hDNA, human DNA.

\section{Figure 3}

ddPCR reaction profiles at higher concentrations of hDNA. A representative reaction profile (A) and graph (B) are shown. NTC, negative template control (no human DNA and $100 \mathrm{ng}$ of rat DNA); hDNA, human DNA. 
Figure 4

A

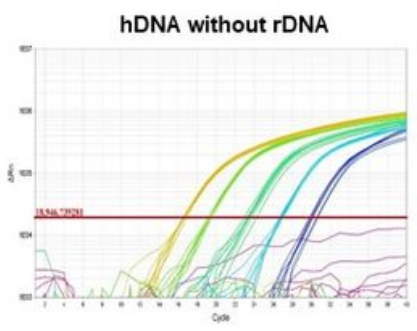

D

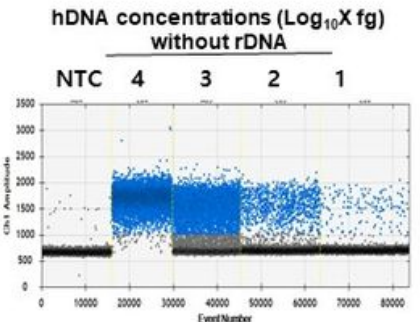

B

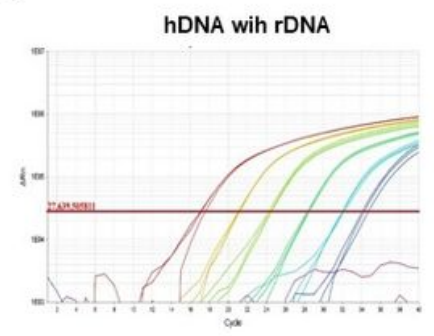

E

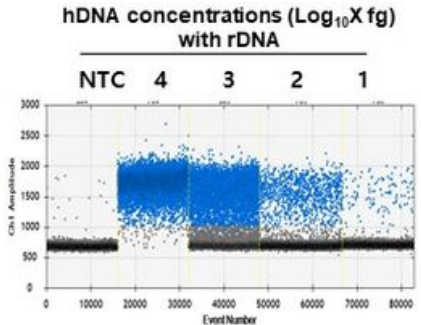

C

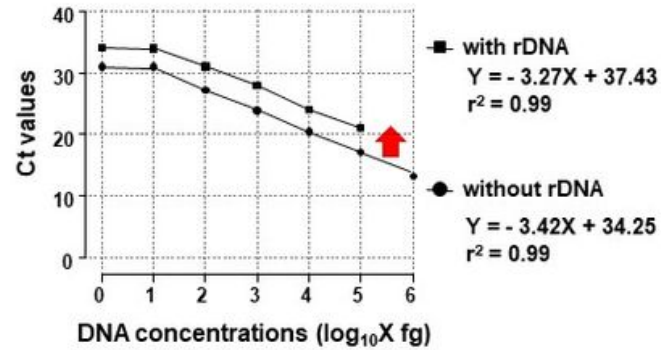

F

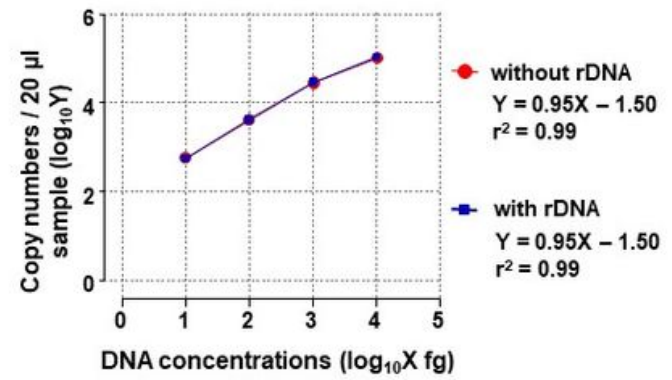

Figure 4

Comparison of the qRT-PCR and ddPCR methods. Human DNA extracted from umbilical cord-derived mesenchymal stem cells was subjected to qRT-PCR to detect Alu elements in the absence (A) or presence (B) of rat DNA. Plotted Ct values for each experiment (C). Similar experiments were performed using ddPCR to detect Alu elements in the absence (D) or presence $(\mathrm{E})$ of rat DNA. The measured copy numbers for each experiment are plotted in (F). NTC, negative template control (no human DNA and $100 \mathrm{ng}$ of rat DNA); hDNA, human DNA; rDNA, rat DNA. 


\section{Figure 5}

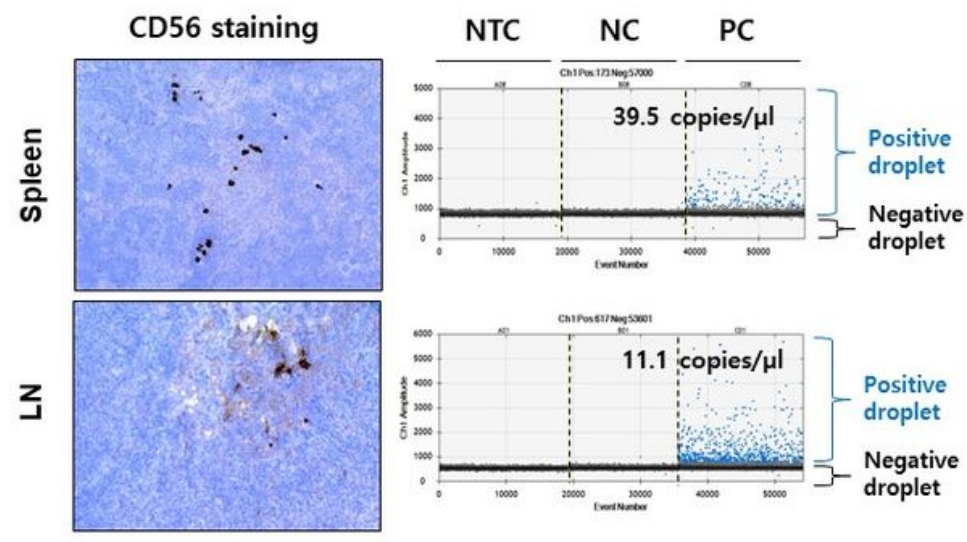

\section{Figure 5}

Ex vivo detection of human natural killer (NK) cells in NOG mice 10 days after injection. Immunohistochemical staining for CD56 showed some residual cells in the spleen (left upper panel) and lymph node (left lower). ddPCR measured 39.5 and 11.1 copies of Alu elements per $5 \mathrm{ng}$ of genomic DNA extracted from the spleen and lymph nodes, respectively. 